\title{
Wechselbarrieren und die
}

\section{Bedeutung spezifischer}

Kassenangebote

\section{Empirische Befunde zum Konsumentenverhalten in der gesetzlichen Krankenversicherung}

Seit Beginn der freien Kassenwahl im Jahr 1996 hat etwa jedes vierte bis fünfte GKV-Mitglied mindestens einmal die Krankenkasse gewechselt. Die meisten Versicherten bleiben ihrer Kasse treu - nur ein Teil davon allerdings aus Überzeugung. Umfragen zufolge weist nur etwa jeder zweite Versicherte eine hohe Kundenbindung auf, der Rest ist als mehr oder weniger wechselgefährdet einzustufen. ${ }^{1}$ Unter den neuen Wettbewerbsbedingungen und den erweiterten Gestaltungsspielräumen in der gesetzlichen Krankenversicherung stellt sich die Frage, warum ein Großteil der Mitglieder entweder noch nie oder schon seit Jahren die Krankenkasse nicht gewechselt hat und welche Faktoren den Verbleib bzw. die Wechselabsicht der GKV-Kunden beeinflussen. Der vorliegende Beitrag fasst hierzu die Ergebnisse der wichtigsten Untersuchungen zur Wechselabsicht der gesetzlich Versicherten sowie zur Bedeutung spezifischer Kassenmerkmale und -angebote zusammen und zeigt offene Forschungsfelder zum Konsumentenverhalten in der gesetzlichen Krankenversicherung auf.

\section{Guido W. Weber}

\section{Untersuchungen zur Wechselabsicht von Versicherten}

Die empirische Forschung zum Konsumentenverhalten in der gesetzlichen Krankenversicherung (GKV) beschäftigte sich in den ersten Jahren der Wahlfreiheit vor allem mit der Gruppe der Kassenwechsler. Die weitaus größere Gruppe der Nicht-Wechsler wurde vergleichsweise wenig beachtet. Insbesondere die Fragen, warum so viele GKV-Kunden ihrer Krankenkasse treu bleiben und welche Faktoren das Ausmaß dieser Treue beeinflussen, blieben über längere Zeit unbeantwortet. Inzwischen liegen erste Studien hierzu vor.

Im Bertelsmann-Gesundheitsmonitor wurde von Höppner et al. (2004) erstmals nach den Gründen für einen

Dipl.-Kfm. Guido W. Weber,

Doktorand am Lehrstuhl für Marketing

Eberhard Karls Universität Tübingen
Nicht-Wechsel der Krankenkasse gefragt. Die Teilnehmer sollten in der Befragung angeben, was sie bei ihrer derzeitigen Krankenkasse hält, welche Bedenken sie gegenüber einer neuen Krankenkasse haben und was sie grundsätzlich an einem Kassenwechsel hindert:

- Gründe für einen Nicht-Wechsel bezogen auf die aktuelle Krankenkasse: GKV-Mitglieder bleiben ihrer Krankenkasse vor allem treu, weil sie sich an ihre Kasse gewöhnt haben (58 Prozent), die Kasse ihnen ein Gefühl von Sicherheit und Verlässlichkeit vermittelt (55 Prozent) oder sie „schon immer“ bei ihrer Krankenkasse versichert sind und damit eine gewisse Tradition verbinden (52 Prozent). Eine untergeordnete Rolle spielen die Solidarität gegenüber den Mitversicherten (13 Prozent) oder die persönliche Bindung zu einem oder mehreren Kassenmitarbeitern (7 Prozent).

- Gründe für einen Nicht-Wechsel bezogen auf eine neue Krankenkasse: Der Gedanke an eine neue Krankenkasse löst bei einem Großteil der Nicht-Wechsler (60 Prozent) 


\section{THEMA}

Ängste und Unsicherheiten aus („man kann nie wissen, ob die neue Krankenkasse nicht doch schlechter ist als die alte“). Schlechte Erfahrungen Dritter (15 Prozent) oder ein fehlendes Vertrauen in eine neue Kasse (14 Prozent) sind für einen Teil der Nicht-Wechsler relevant. Unkenntnis in Bezug auf den Kontrahierungszwang der Kassen („ich kann mir nicht vorstellen, dass mich eine neue Krankenkasse aufnehmen würde") hält nur wenige Mitglieder (9 Prozent) von einem Wechsel ab. Ein Prozent der Nicht-Wechsler wurde von einem Kassenmitarbeiter vor einem Wechsel gewarnt.

Grundsätzliche Hindernisse für einen Wechsel der Krankenkasse: Fast jeder zweite Nicht-Wechsler (46 Prozent) ist an einem Kassenwechsel prinzipiell nicht interessiert. Jeder Vierte (24 Prozent) nimmt keine Unterschiede zwischen den Krankenkassen wahr. Administrative Wechselkosten (25 Prozent), Zeitmangel (22 Prozent) und die Unkenntnis von Informationsquellen (11 Prozent) stellen weitere Hindernisse für einen Kassenwechsel dar. Auch einen Vergleich der verschiedenen Krankenkassen empfindet ein Viertel (24 Prozent) der Nicht-Wechsler als zu schwierig.

Folgt man den Befragungsergebnissen, dann sind die wesentlichen Motive für einen Kassenverbleib die Gewöhnung und positive Erfahrungen („Sicherheit und Verlässlichkeit“) sowie ein Traditionsbewusstsein der Mitglieder. Darüber hinaus spielen Ängste und Unsicherheiten gegenüber neuen Anbietern sowie administrative und psychische Wechselkosten eine Rolle.

Braun et al. (2006) nehmen eine tiefergehende Analyse der Wechselbarrieren in der gesetzlichen Krankenversicherung vor. Grundlage sind die Daten einer im Winter 2005/2006 durchgeführten Zusatzbefragung von Teilnehmern des Gesundheitsmonitors. Die Autoren unterscheiden sechs Faktoren, die den emotionalen und faktischen Barrieren („Verbundenheit“ bzw. „Gebundenheit“) eines Anbieterwechsels entsprechen. ${ }^{2}$ Die Ergebnisse ihrer Studie werden auch in anderen Untersuchungen weitgehend bestätigt:

Zufriedenheit mit der Krankenkasse: Wie in anderen Märkten, so zeigen auch Untersuchungen in der GKV, dass überdurchschnittlich zufriedene Kunden eine deutlich geringere Wechselabsicht in Umfragen angeben als weniger zufriedene Kunden. ${ }^{3}$ Auch in der Studie von Braun et al. (2006) erweist sich die Zufriedenheit „als ein ganz zentraler Einflussfaktor auf alle Indikatoren der Wechselneigung". ${ }^{4}$ Je nach Erhebung werden allerdings unterschiedliche Zufriedenheitswerte ermittelt: Bei Braun et al. (2006) geben gut 80 Prozent der Versicherten an, mit ihrer Krankenkasse (eher, sehr oder vollkommen) zufrieden zu sein. Schaaf/Sander (2008) identifizieren 56 Prozent „überdurchschnittlich zufriedene" Versicherte. ${ }^{5}$ Im BKK Imagemonitor 2008 sind 63 Prozent der Befragten „allgemein zufrieden“. ${ }^{6} \mathrm{Im}$ WIdO-monitor 2009 liegt der Anteil der „zufriedenen“ und „sehr zufriedenen “ Versicherten bei 69 Prozent, ${ }^{7}$ im M+M Versichertenbarometer 2009 fallen 92 Prozent der Befragten in diese Kategorien. ${ }^{8}$ Aus einer Erhebung der
Ratingagentur ASSEKURATA vom Februar 2009 gehen etwa 50 Prozent „zufriedene“, 30 Prozent „sehr zufriedene“ und 12 Prozent „vollkommen zufriedene“ Versicherte hervor. ${ }^{9}$ Neben diesen Momentaufnahmen stellt Meyer (2009) fest, dass „die allgemeine Zufriedenheit bei den Mitgliedern aller Kassen in den letzten Jahren mehr oder weniger konstant gesunken" ist und mittlerweile „keine Kasse mehr einen Wert von zwei Dritteln oder mehr allgemein zufriedener Mitglieder " erreicht. ${ }^{10}$ Die Zufriedenheit kann in vielen Fällen eine Wechselbarriere für GKV-Kunden darstellen, sie ist aber auch in diesem Markt weder eine notwendige noch eine hinreichende Bedingung dafür, dass Versicherte bei ihrer Krankenkasse bleiben. Auch sehr zufriedene Mitglieder weisen zum Teil eine hohe Wechselbereitschaft auf; in der Studie der ASSEKURATA trifft dies für acht Prozent der befragten GKV-Kunden zu. ${ }^{11}$ Schaaf/Sander (2008) stufen jeden zweiten überdurchschnittlich zufriedenen Versicherten als latent oder akut wechselgefährdet ein. ${ }^{12}$

Fehlende Verbesserungschancen aufgrund geringer Kassenunterschiede: In der Untersuchung von Braun et al. (2006) bewertet jeder vierte Befragte (23 Prozent) die Angebote von Krankenkassen als uniform, der Rest nimmt Unterschiede wahr. ${ }^{13}$ Hierzu zählen vor allem die Kulanz bei Leistungsbewilligungen (64 Prozent), der Beitragssatz (45 Prozent) sowie generelle Leistungsdifferenzen (36 Prozent). Versicherte, die Unterschiede zwischen den Kassen wahrnehmen, neigen eher zu einem Kassenwechsel. Einen signifikanten Zusammenhang stellen Braun et al. (2006) mithilfe einer logistischen Regression fest. Auch im WIdO-monitor 2006 wurde das Desinteresse an einem Kassenwechsel von den Befragten u.a. damit begründet, dass sich die Krankenkassen „in ihren Leistungen und Beiträgen letztendlich nicht unterscheiden “. ${ }^{14}$ Nach einer aktuellen Studie der dostal $\&$ partner management-beratung gmbh konnten im Winter 2007/2008 tatsächlich nur etwa 60 Prozent der Befragten Unterschiede zwischen den Krankenkassen erkennen. Ein Jahr später (bei der Einführung des Gesundheitsfonds und des einheitlichen Beitragssatzes) waren es nur noch rund 40 Prozent. Mehr als einen Unterschied nimmt nur etwa jeder sechste Versicherte wahr. ${ }^{15} \mathrm{Zu}$ den angegebenen Unterscheidungsmerkmalen gehören v.a. der Beitragssatz bzw. die Kosten (56 Prozent in beiden Erhebungswellen) sowie die Leistungen der Krankenkassen (2007/2008: 28 Prozent; 2008/2009: 59 Prozent). Andere Aspekte (wie z.B. die Kompetenz, der Service oder das Image) wurden deutlich seltener genannt. ${ }^{16} \mathrm{Zu}$ ähnlichen Ergebnissen kommt die Frühjahrsbefragung 2009 des Gesundheitsmonitors. Demnach bestehen für 43 Prozent der Versicherten deutliche Unterschiede zwischen den Leistungen und den Services der Krankenkassen, für den Rest sind die Angebote nahezu gleich. ${ }^{17}$ Neben fehlenden Verbesserungschancen kann auch die Angst vor einer Verschlechterung die Wechselbereitschaft verringern. So befürchten nach einer Erhebung von Korehnke Kommunikation (2009) 38 Prozent der Nicht-Wechsler, dass sich ein Kassenwechsel negativ auf ihre medizinische Versorgung auswirkt. ${ }^{18}$ 
Unterschätzung von Einsparmöglichkeiten: Über die Hälfte der Versicherten hat nach Braun et al. (2006) keine Vorstellung davon, ob sich ein Kassenwechsel finanziell lohnt bzw. schätzt die Einsparmöglichkeiten als gering (unter 10 Euro pro Monat) ein. ${ }^{19}$ Zwischen der Höhe der vermuteten Einsparungen und der Wechselneigung der Versicherten besteht ein signifikanter Zusammenhang, d.h. GKV-Kunden, die sich von einem Kassenwechsel einen finanziellen Vorteil versprechen, weisen eine höhere Wechselbereitschaft auf. Die Unkenntnis von Einsparmöglichkeiten stellt dagegen eine Wechselbarriere dar. Ähnliche Ergebnisse gehen auch aus der Continentale-Studie aus dem Jahr 2002 hervor: ${ }^{20}$ In der Befragung konnte die eine Hälfte der Versicherten überhaupt keine Angaben über das Einsparungspotenzial bei einem Kassenwechsel machen. Von der anderen Hälfte gaben immerhin 35 Prozent (also rund 18 Prozent der Versicherten, die insgesamt befragt wurden) eine realistische Einsparung von unter 40 Euro pro Monat an; ein Wechsel der Krankenkasse kam bei den Befragten allerdings erst ab einer durchschnittlichen Einsparung von 47 Euro pro Monat infrage. Die Verfasser der Studie halten daher fest: „Die Menschen glauben zwar, sie würden durch einen Wechsel der Krankenkasse Geld sparen. Die Ersparnis wird aber nicht für hoch genug gehalten, um einen Anreiz zum Wechsel der Krankenkasse zu bieten. “21 Felder (2007) weist darauf hin, dass der Beitragssatz für ein Drittel der etwa 70 Millionen GKVVersicherten überhaupt keine Rolle spielt, da fast 19 Millionen Versicherte eine kostenfreie Familienversicherung in Anspruch nehmen und die Beiträge für die rund fünf Millionen Bezieher von Arbeitslosengeld II der Bund übernimmt. ${ }^{22}$ Darüber hinaus zeigt Felder (2007), dass die Opportunitätskosten eines Nicht-Wechsels für viele Versicherte vor der Einführung des einheitlichen Beitragssatzes in der Tat gering waren: Bei einem Beitragssatzunterschied von zwei bzw. drei Prozentpunkten hätten im Jahr 2003 etwa 17 bzw. 11 Millionen Versicherte einen finanziellen Nachteil von maximal zehn Euro pro Monat erlitten. Zählt man die Familienversicherten und die Bezieher von Arbeitslosengeld II hinzu, dann verzichteten etwa die Hälfte der GKV-Versicherten bei einem Nicht-Wechsel ihrer Krankenkasse auf einen monatlichen Betrag von höchstens zehn Euro. ${ }^{23}$ Inzwischen gelten neue Regeln. Unter dem einheitlichen Beitragssatz spielt sich der Preiswettbewerb der Krankenkassen zukünftig auf der Ebene der Zusatzbeiträge und Ausschüttungen ab. In einer Untersuchung des BKK Bundesverbands vom Frühjahr 2008 gaben 35 Prozent der Befragten an, bei der Erhebung eines Zusatzbeitrags ihre Krankenkasse wahrscheinlich bzw. sicher wechseln zu wollen. ${ }^{24}$ Korehnke Kommunikation (2009) kommen ein Jahr später auf eine Quote von 42 Prozent. ${ }^{25}$ Bei einer Ausschüttung von 10 Euro im Monat durch eine andere Krankenkasse zogen 27 Prozent der Befragten in der BKK-Studie sicher oder wahrscheinlich einen Kassenwechsel in Betracht, bei einem Betrag von 20 Euro traf dies bereits für jeden zweiten Befragten zu. Sowohl bei Zusatzbeiträgen als auch bei Ausschüttungen zeigten Versicherte, die schon länger bei ihrer Kasse versichert waren und eine hohe Zufriedenheit mit dem Leistungsangebot und dem Service äußerten, eine deutlich geringere Wechselbereitschaft als unzufriedene Versicherte und Versicherte mit einer Mitgliedschaftsdauer von nur ein bis zwei Jahren.

- Loyalität zur Krankenkasse: Jeder fünfte Befragte gibt bei Braun et al. (2006) eine persönliche Bindung zu einem oder mehreren Kassenmitarbeitern an. ${ }^{26}$ Die Wechselneigung wird hiervon jedoch nicht beeinflusst, ein entsprechender Effekt lässt sich aus den Ergebnissen einer multivariaten Analyse nicht ableiten. Gesetzlich Versicherte treten auch eher selten in Kontakt mit ihrer Krankenkasse. Laut dem M+M Versichertenbarometer 2009 hatte nur etwas mehr als die Hälfte der GKVKunden innerhalb des vorangegangenen Jahres mindestens einmal Kontakt zu ihrer Kasse, bei jedem vierten Versicherten waren es drei oder mehr Kontakte. ${ }^{27}$ Ein persönlicher Kontakt über die Geschäftsstelle ist nur 28 Prozent der Versicherten wichtig. Der Rest der Versicherten bevorzugt das Telefon (45,6 Prozent), Briefe und Telefax (22,6 Prozent) oder das Internet und E-Mail (2,8 Prozent). ${ }^{28}$

- Scheu vor dem Wechsel-Aufwand: Etwa die Hälfte der GKV-Kunden empfindet einen Kassenwechsel als „sehr anstrengend und lästig“, für 70 Prozent der Versicherten ist es zudem „sehr mühsam und zeitaufwendig“, „sich vor einem Kassenwechsel zu informieren“. ${ }^{29}$ Trotz dieser hohen Werte können Braun et al. (2006) keinen signifikanten Zusammenhang mit der Wechselneigung feststellen. Administrative und psychische Wechselkosten sind demnach keine Barrieren für einen Kassenwechsel.

- Wahrgenommenes Risiko von Wechsel-Sanktionen: Irrtümer über die rechtlichen Rahmenbedingungen eines Kassenwechsels (z.B. der Verlust angesparter Beiträge, höhere Prämien für Ältere oder die Ablehnung einer Kassenmitgliedschaft) liegen bei vielen Versicherten vor und stellen nach Braun et al. (2006) eine überaus bedeutsame Wechselbarriere dar. ${ }^{30}$ Bei Versicherten, die eher unzufrieden mit ihrer Krankenkasse sind, schwankt die Wechselneigung um bis zu 40 Prozentpunkte. Der Wert hängt davon ab, ob bzw. wie viele Irrtümer vorliegen. ${ }^{31}$

Die meisten GKV-Kunden sind mit ihrem Anbieter grundsätzlich zufrieden, d.h. die Erwartungen der Versicherten werden von den Kassen weitgehend erfüllt. Nur wenige Mitglieder verspüren daher den Drang, ihre Krankenkasse aufgrund von Unzufriedenheit wechseln zu wollen, allerdings nehmen auch erst wenige Versicherte relevante Unterschiede zwischen den Krankenkassen wahr. Dies kann unter den eingeschränkten Handlungsspielräumen und den unausgereiften Anreizstrukturen, die über viele Jahre den Wettbewerb der Krankenkassen prägten und behinderten, nicht verwundern.

Mit einem Kassenwechsel ließ sich in den ersten Jahren der Wahlfreiheit vor allem Geld sparen. Die Höhe der möglichen Ersparnis war für einen Großteil der Versicherten allerdings gering und reichte in vielen Fällen als Wechsel- 


\section{THEMA}

anreiz vermutlich nicht aus. Nur Gutverdiener konnten vor der Einführung des einheitlichen Beitragssatzes im Jahr 2009 von einem Kassenwechsel spürbar finanziell profitieren. Eine Verbesserung der medizinischen Versorgung oder die Befriedigung individueller Bedürfnisse durch entsprechende Zusatzangebote waren über einen Wechsel der Kasse dagegen kaum möglich. Viele Versicherte empfinden einen Wechsel zudem als anstrengend und mühsam. Braun et al. (2006) stellen zwar keinen signifikanten Einfluss der Wechselkosten auf die Wechselbereitschaft der Versicherten fest, denkbar ist jedoch, dass sich Krankenkassen erst stärker differenzieren müssen, bevor die Wechselkosten in die Entscheidung der Versicherten eingehen. Solange das allgemeine Desinteresse an einem Kassenwechsel überwiegt, spielt der Aufwand für einen Wechsel vermutlich nur eine untergeordnete Rolle. Auch die immer noch weit verbreiteten Irrtümer über WechselSanktionen können vor allem unzufriedene Kunden von einem Wechsel abhalten. Über eine bessere Aufklärung ließe sich in diesem Punkt Abhilfe schaffen und die Dynamik des Kassenwettbewerbs erhöhen.

Mit dem GKV-Wettbewerbsstärkungsgesetz (2007) wurden die Handlungsmöglichkeiten der Krankenkassen erweitert und neue Anreize für den Aufbau innovativer Versorgungsstrukturen geschaffen. Unter diesen Rahmenbedingungen ist zu erwarten, dass sich die Angebote der Kassen zukünftig stärker voneinander unterscheiden werden als dies bislang der Fall war. Um in den „richtigen“ Bereichen aktiv zu werden, müssen Krankenkassen jedoch wissen, welche Angebote den Wünschen und Bedürfnissen der Versicherten entsprechen.

Noch im Sondergutachten 1995 bemängelte der Sachverständigenrat, dass zu den Präferenzen der Versicherten erst vereinzelte Befragungen vorliegen. ${ }^{32}$ Mittlerweile kann auf mehrere empirische Befunde zurückgegriffen werden.

\section{Die Bedeutung spezifischer Kassenangebote}

Eine hohe Kundenzufriedenheit und -bindung setzen voraus, dass Anbieter die Erwartungen ihrer Kunden besser erfüllen als die Konkurrenz. Für Krankenkassen stellt sich somit die Frage, was Versicherte von ihnen erwarten, wie wichtig spezifische Merkmale und Angebote für die Versicherten sind, welcher Nutzen von diesen Angeboten ausgeht und wie hoch die Zahlungsbereitschaft hierfür ist.

\subsection{Erwartungen an eine Krankenkasse und Wichtigkeit spezifischer Merkmale und Angebote}

Über lange Zeit waren Krankenkassen „nicht viel mehr als die Inkasso- und Distributionsabteilung der Ärzteschaft“. ${ }^{33}$ Dieses Bild hat sich in den letzten Jahren grundlegend gewandelt. Aus den einstigen „Verwaltern“ werden zunehmend „Gestalter“. Spätestens seit der Einführung der freien Kassenwahl bemühen sich Krankenkassen darum, nicht mehr nur „Payer“ im Gesundheitswesen zu sein, sondern die Rolle von aktiven „Playern“ und kundenorientierten Dienstleistern einzunehmen. Dieses neue Selbstverständnis spiegelt sich auch in der Wahrnehmung und den Erwartungen der Versicherten wider:

Zok (2002) untersucht auf Basis mehrerer Repräsentativumfragen aus den Jahren 1998-2001, welches Versicherungsverständnis GKV-Mitglieder haben. Demnach wünscht sich ein Großteil der Versicherten eine aktivere Rolle der Krankenkassen bei der Information, Aufklärung und Betreuung von Patienten. Gleiches gilt für die Gesundheitsförderung und Prävention. Darüber hinaus stimmen die Mitglieder zu, dass sich Krankenkassen um eine Verbesserung und Erneuerung der Versorgungsstrukturen bemühen sollten. Dem Thema „Selektivverträge“ stehen die meisten Versicherten ebenfalls offen gegenüber. Krankenkassen werden demnach nicht mehr nur als der „zahlende Dritte“, sondern zunehmend als „Intermediär zwischen Versicherten und Leistungserbringern" gesehen; eigene ärztliche Leistungen sollten Krankenkassen nach Meinung der Befragten allerdings eher nicht erbringen. Das Anforderungsprofil der Krankenkassen verändert sich somit deutlich in Richtung Beratungs-, Betreuungs- und Koordinationsfunktion.

- Im Vorfeld der Gesundheitsreform 2003/2004 wurden auch Versicherte einer Ortskrankenkasse im Ruhrgebiet zu ihrem Funktionsverständnis der Krankenkasse befragt. Dabei beschrieben 40 Prozent der Teilnehmer die AOK als „Dienstleistungsunternehmen“ und 58 Prozent als „Gesundheitsinstitution“ (Mehrfachnennungen waren möglich). Nur wenige Kunden sahen in ihrer Kasse eine „Verwaltungseinrichtung“ (12 Prozent) oder „Behörde“ (11 Prozent). ${ }^{34}$ Trotz dieser eindeutigen Charakterisierung glaubten jedoch nur 28 Prozent der Befragten, „dass sich die AOK im Vergleich zu früher intensiver um die Belange ihrer Versicherten kümmert“; von den Mitarbeitern vertraten fast 80 Prozent diese Meinung. ${ }^{35}$ Das Bild einer kundenfernen Verwaltungsbehörde ist in den Köpfen der Versicherten demnach fast verschwunden, das von den Mitarbeitern empfundene Engagement erreichte zum Zeitpunkt der Befragung aber erst wenige Versicherte. Die Rolle als „Gestalter" und medizinischer Begleiter wird von einem Teil der AOK-Kunden akzeptiert. Auf die Frage, „womit die AOK bei den Versicherten Aufmerksamkeit erzeugen“ kann, gab fast jeder zweite Versicherte (49 Prozent) die „medizinische Fachkompetenz“ der Kasse an. Deutlich weniger Kunden nannten „Informationen für Versicherte“ (29 Prozent), „Gesundheitskurse“ (15 Prozent) oder „ansprechende Räume“ (acht Prozent). ${ }^{36}$

Auch aus einer repräsentativen Befragung der Unternehmensberatung Roland Berger, die im Mai 2008 bei 1.000 Erwachsenen durchgeführt wurde, geht das neue Profil von Krankenkassen hervor. So soll aus Sicht der Befragten „die Krankenversicherung keinesfalls auf ihre Rolle als Kostenträger beschränkt bleiben, sondern aktiv ins Geschehen eingreifen können“". ${ }^{37}$

$\mathrm{Zu}$ ähnlichen Ergebnissen kommt der WIdO-monitor 2009: Die Aussagen von 3.000 GKV-Versicherten bestä- 
tigen ein weiteres Mal, dass Krankenkassen „nicht mehr nur als Beitragseinzugsstelle wahrgenommen werden, sondern zunehmend von ihnen auch eine aktive Rolle bei der Koordination von Gesundheitsleistungen erwartet wird“. ${ }^{38}$ Den Versicherten ist u.a. wichtig, dass die Krankenkasse sie in ihren Rechten unterstützt, chronisch Kranke intensiv betreut, die Qualität und Abrechnungen der Leistungserbringer überprüft, die Versicherten in gesundheitsbezogenen Fragen informiert und berät, Zusatzleistungen anbietet und bei der Verbesserung der medizinischen Versorgung eine aktive, gestaltende Rolle übernimmt. Ein Großteil der befragten GKV-Versicherten stimmte den meisten dieser Punkte $\mathrm{Zu}^{39}$

Abhängig von der jeweiligen Fragestellung und den vorgegebenen Antwortmöglichkeiten rücken neben den Beitrags-, Leistungs- und Serviceaspekten auch andere, allgemeine Dienstleistungsmerkmale von Krankenkassen in den Vordergrund. Hierzu gehören z.B. eine fachkundige Beratung, die Freundlichkeit und Hilfsbereitschaft der Mitarbeiter, die Zuverlässigkeit der Kasse, eine schnelle Kostenregulierung, die pünktliche Auszahlung von Geldleistungen, eine gute telefonische Erreichbarkeit sowie verständliche und übersichtliche Formulare. Folgt man dem M+M Versichertenbarometer 2009, dann nehmen diese Merkmale sogar „den höchsten Stellenwert in den Erwartungen der Versicherten über alle Kriterien ein. Zum Teil liegen die von den Versicherten angegebenen Wichtigkeiten deutlich über den so genannten „harten“ Faktoren „Leistungen und Beiträge“, die häufig als Wechselmotive genannt werden." 40

Auch in Befragungen des stern, des BKK Bundesverbands und des Gesundheitsmonitors stehen „soft facts“, wie z.B. eine gute telefonische Erreichbarkeit und eine schnelle Kostenregulierung, z.T. auf gleicher Ebene oder sogar über dem Preis-Leistungsverhältnis, günstigen Tarifen und einem umfangreichen Leistungsangebot der Krankenkassen - zumindest gibt ein Großteil der Versicherten an, dass diese Kriterien besonders wichtig für sie sind. Deutlich weniger wichtig stufen GKV-Kunden spezielle Versorgungsangebote, eine Geschäftsstelle in der Nähe, interessante Beitragsmodelle und Bonusprogramme, umfangreiche schriftliche Informationen, ein ansprechendes Internetangebot, eine (kostenlose) 24-Stunden-Hotline oder die Unterstützung bei der Vermittlung von Arztterminen ein. ${ }^{41}$

Menschen sind tendenziell „Anspruchs-Maximierer“, d.h. sie fordern von einem Produkt oder Dienstleistungsanbieter optimale Leistung. Vieles ist aus ihrer Sicht gleich wichtig oder unverzichtbar. Werden Kunden nach der Wichtigkeit einzelner Merkmale gefragt, so geben sie entgegen ihrem tatsächlichen Empfinden oftmals höhere Wichtigkeiten an. Die Antworten konzentrieren sich zumeist am oberen Ende der Bewertungsskala. ${ }^{42}$ Aufgrund dieser „Anspruchsinflation“ ist es nicht zweckmäßig, die Wichtigkeit von Leistungsmerkmalen direkt abzufragen. Die so gewonnenen Ergebnisse sind i.d.R. verzerrt und können zu falschen Handlungsempfehlungen führen. Folgt man darüber hinaus dem Mehr-Faktoren-Modell von
Kano, dann hängt die Wichtigkeit eines Merkmals auch davon ab, ob es sich um einen Basis-, Leistungs- oder Begeisterungsfaktor handelt. ${ }^{43}$ Auf Basis der bisherigen Untersuchungen bleibt demnach offen, welche Bedeutung den genannten Kassenmerkmalen und -angeboten tatsächlich zukommt und wie diese das Verhalten der Versicherten beeinflussen.

Eine Ausnahme stellen die Ergebnisse der ASSEKURATAStudie aus dem Frühjahr 2009 dar: Um mehr über die Wichtigkeit einzelner Kassenmerkmale und -angebote herauszufinden, wurde überprüft, in welchem Maße die wahrgenommenen Vorteile der eigenen Krankenkasse mit dem Kundenbindungsindex der Versicherten korrelieren. Demnach „haben hilfreiche Informationsangebote und altersunabhängige Versorgungsangebote den größten Einfluss auf die Kundenbindung bei den untersuchten Krankenkassen.“ Dagegen „wirken Prämien für die Nichtinanspruchnahme von Leistungen und die Möglichkeit eines persönlichen Gesprächs in der Geschäftsstelle vergleichsweise gering auf die Bindung der Versicherten. “44

Um die Wichtigkeit von Leistungsmerkmalen zu bestimmen, können unterschiedliche Verfahren herangezogen werden. Neben der Berechnung von Korrelationen lässt sich auch der Nutzen einzelner Merkmale ermitteln.

\subsection{Nutzen spezifischer Kassenangebote und Zahlungsbereitschaft der Versicherten}

Produkte und Dienste stellen i.d.R. ein Bündel von Eigenschaften dar, die für die Nachfrager einen unterschiedlichen Teilnutzen haben. Wird ein Angebot modifiziert, indem man z.B. Eigenschaften hinzufügt oder entfernt, so verändert sich der Gesamtnutzen des Angebots für die Kunden. Diese Nutzenveränderungen sind nicht nur bei gesundheitspolitischen Reformvorhaben sondern auch bei der kassenindividuellen Gestaltung von Leistungs- und Serviceangeboten von Interesse, geben sie doch die Präferenzen der Versicherten unmittelbar wieder.

Um den Teilnutzen einzelner Kassenmerkmale zu bestimmen, wird in den Arbeiten von Nolting et al. (2006), Becker/Brändle/Zweifel (2007) und Greß et al. (2008b) auf ein so genanntes Discrete-Choice-Experiment - eine Variante des Conjoint-Verfahrens ${ }^{45}$ - zurückgegriffen. Die Studie von Scheffold (2008) basiert auf einer Limit ConjointAnalyse. In beiden Fällen müssen die Teilnehmer einen Paarvergleich durchführen. Man präsentiert ihnen hierzu zwei unterschiedliche Angebote und lässt sie anschließend entscheiden, welches Angebot sie vorziehen; in der Limit Conjoint-Analyse ist es zudem möglich, beide Angebote abzulehnen (Nichtkaufentscheidung). Wird neben den Leistungsattributen auch der Preis für das jeweilige Angebot angegeben, so kann aus den Befragungsergebnissen der Nutzen eines Merkmals in Geldeinheiten bestimmt werden. Die Ergebnisse der vier Studien geben Aufschluss darüber, wie wichtig spezifische Kassenmerkmale für die Versicherten sind: 


\section{THEMA}

Das erste Discrete-Choice-Experiment in der gesetzlichen Krankenversicherung fand im Frühjahr 2005 als Teil der 4. Delphi-Studie zur Zukunft des Gesundheitswesens statt. ${ }^{46}$ Nolting et al. (2006) untersuchten dabei, welchen Wert die Versicherten einzelnen Elementen des GKV-Leistungskatalogs beimessen. Zu den analysierten Merkmalen gehörten die freie Arztwahl, die Einschränkung der Auswahl an verschreibungspflichtigen Arzneimitteln, die Steuerung der Krankenhausbehandlung bei planbaren Behandlungen sowie die Einschränkung oder Erweiterung des Zugangs zu therapeutischen Innovationen. Während sich die ersten drei Merkmale auf konkrete Reformvorhaben bezogen, stellte das vierte Merkmal eine fiktive Reformoption dar. In der Untersuchung mussten die Teilnehmer den Status quo der gesetzlichen Krankenversicherung mit alternativen Versicherungsangeboten vergleichen und ein Präferenzurteil abgeben. Hierzu wurde der Preis bzw. eine Preisminderung als fünftes Merkmal aufgenommen, da jedes der Alternativangebote (mit Ausnahme des erweiterten Zugangs zu Innovationen) eine Einschränkung gegenüber den aktuellen GKV-Regelungen darstellte. Um die Versicherten zu einem fiktiven Anbieterwechsel zu bewegen, musste dem jeweiligen Nutzenverlust ein entsprechender finanzieller Ausgleich entgegenstehen. Über ein Viertel (27,5 Prozent) der Befragten entschied sich trotz erheblicher Beitragsminderungen stets für den Status quo, die restlichen Teilnehmer bevorzugten mindestens eine der angegebenen Alternativen. Der höchste durchschnittliche Kundennutzen wurde für die freie Arztwahl festgestellt. Erst ab einem Beitragsnachlass von 260 Euro pro Jahr war die Hälfte der Befragten bereit, auf dieses Merkmal zu verzichten. Dahinter folgten der offene Zugang zu Innovationen (ca. 125 Euro), die freie Auswahl an verschreibungspflichtigen Arzneimitteln (ca. 120 Euro) und die freie Auswahl eines Krankenhauses (ca. 100 Euro). Der durchschnittliche Nutzen für den erweiterten Zugang zu Innovationen lag bei 125 Euro pro Jahr. Je nach Untergruppe wichen die Kompensationsforderungen und Zahlungsbereitschaften deutlich von den durchschnittlichen Beträgen ab. Neben geschlechts- und bildungsspezifischen Unterschieden spielte für die Bewertung der Merkmale vor allem die Inanspruchnahme medizinischer Leistungen (und somit der Gesundheitszustand der Versicherten) eine Rolle. Im Experiment hätte die Hälfte der Versicherten mit einer stationären Behandlung im letzten Jahr erst ab einem Beitragsnachlass von 82,7 Prozent auf die freie Arztwahl verzichtet; über alle Befragten lag dieser Wert bei 17,6 Prozent (dies entspricht den zuvor genannten 260 Euro $^{47}$ ).

Im Mittelpunkt der von Becker/Brändle/Zweifel (2007) durchgeführten Untersuchung stehen neue Modelle der ambulanten Versorgung. ${ }^{48}$ Die Teilnehmer wurden auch in dieser Studie gebeten, den Status quo der GKV mit hypothetischen Versicherungsverträgen paarweise zu vergleichen und sich für den aus ihrer Sicht besten Vertrag zu entscheiden. In den fiktiven Versicherungsverträgen wurden fünf Eigenschaften in unterschiedli- chen Ausprägungen kombiniert: Die Art der ärztlichen Versorgung (freie Arztwahl, Ärzteliste, Hausarztmodell, Netzwerk von Ärzten), das Einholen einer Zweitmeinung (Praxisgebühr ohne Überweisungsschein, Einholen einer Zweitmeinung ohne Zuzahlung), Zusatzleistungen der Krankenkasse (kein besonderes Service- und Informationsangebot, Patientenberater in der Krankenkasse), das Anreizsystem (kein besonderes Anreizsystem, Beitragsrückerstattung bei Nichtinanspruchnahme, Kostenbeteiligung, Bonus für gesundheitsbewusstes Verhalten) sowie die Erhöhung bzw. Senkung des Kassenbeitrags (+/- 200, 300, 400, 500 Euro jährlich). Ausgehend vom Status quo wählten 80 Prozent der Befragten mindestens einen alternativen Versicherungsvertrag. Die durchschnittlichen Zahlungsbereitschaften und Kompensationsforderungen für einzelne Eigenschaften können Abbildung 1 entnommen werden. Den höchsten Wert erreichte erneut die freie Arztwahl. In den sozioökonomischen Untergruppen zeigten die Ergebnisse wiederum erhebliche Unterschiede. Je nach Alter, Geschlecht und Gesundheitszustand haben die Versicherten überaus heterogene Präferenzen.

Die Arbeit von Greß et al. (2008b) geht ebenfalls auf die Leistungs- und Servicekomponenten der Krankenkassen ein..$^{49}$ Die im Paarvergleich zur Auswahl gestellten Versicherungsverträge unterschieden sich in sechs Merkmalen: Der Erreichbarkeit (Telefon, Brief, Internet vs. Geschäftsstelle in Wohnortnähe), dem Informationsservice (besonderer Informationsservice vs. kein spezieller Informationsservice), dem Leistungsangebot (zusätzliche Versorgungsangebote vs. gesetzlicher Versorgungsumfang), der Wahlfreiheit (Auswahl nur bestimmter Leistungserbringer vs. freie Wahl von Ärzten und Kliniken), der Möglichkeit einer Beitragsreduktion (finanzielle Belohnung bei geringer Inanspruchnahme vs. keine finanzielle Belohnung) sowie dem Beitragssatz (12,0\% vs. 13,5\% vs. 15,0\%). 63 Prozent der Befragten waren in dem Experiment bereit, ihren Status quo gegen mindestens eine der vorgelegten Alternativen aufzugeben. Den durchschnittlich ermittelten Teilnutzen der einzelnen Merkmale über alle Befragten zeigt Tabelle 1. Auch diese Werte weichen in den spezifischen Untergruppen zum Teil erheblich ab.

Tabelle 1: Teilnutzenwerte der Kassenmerkmale in Beitragssatzpunkten (Quelle: Greß et al. (2008b), S.67)

\begin{tabular}{|l|l|}
\hline Merkmal & Teilnutzen \\
\hline Geschäftsstelle in Wohnortnähe & 1,60 \\
\hline Besonderer Informationsservice & 0,73 \\
\hline Zusätzliche Versorgungsangebote & 1,07 \\
\hline Freie Arzt- und Klinikwahl & 2,34 \\
\hline Finanzielle Belohnung bei wenig Arztbesuchen & 0,62 \\
\hline
\end{tabular}

Scheffold (2008) widmet sich in ihrer Arbeit den möglichen Auswirkungen des Vertragswettbewerbs in der GKV. ${ }^{50}$ Sollte der Sicherstellungsauftrag für die medizinische Versorgung eines Tages komplett auf die Kran- 
Abbildung 1: Durchschnittliche Zahlungsbereitschaften und Kompensationsforderungen für einzelne Eigenschaften neuer Versorgungsmodelle in Euro pro Jahr (Quelle: Becker/ Zweifel (2006), S.289)

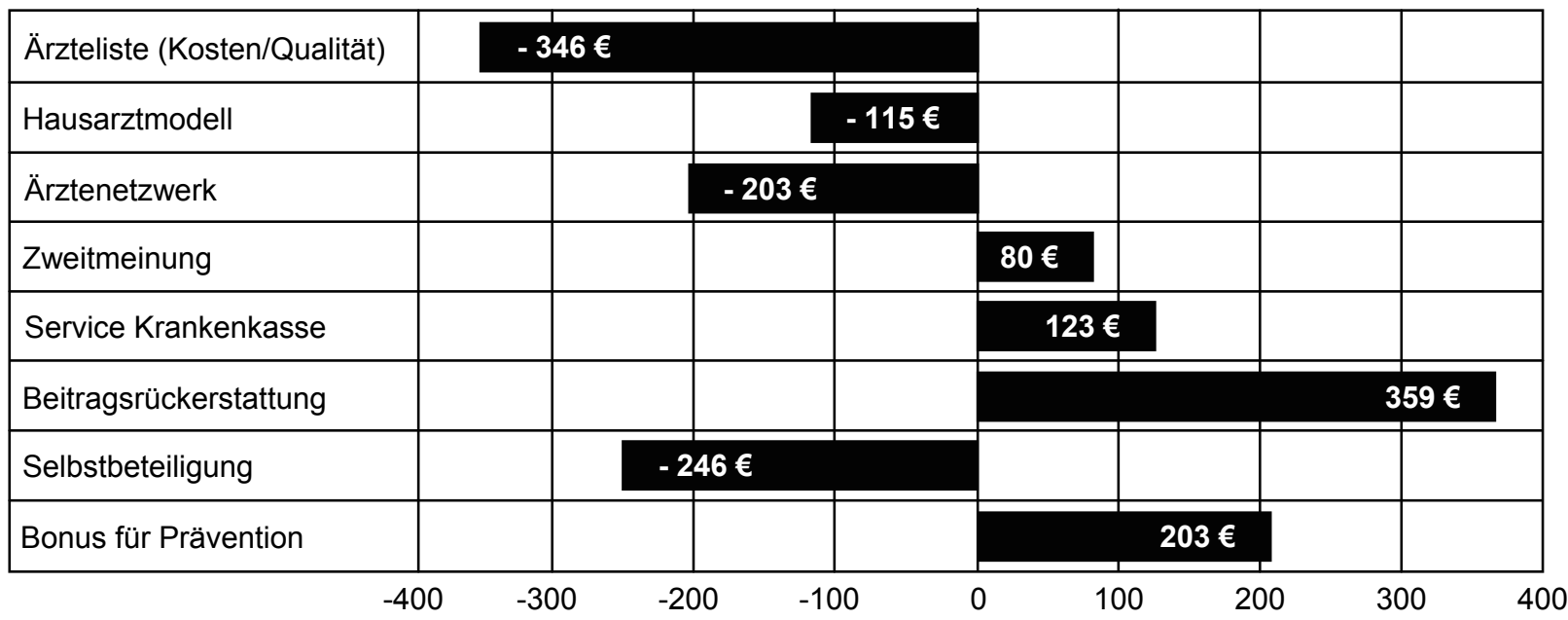

kenkassen übergehen, so stellt sie die Frage, wie wichtig die Qualität und die Anzahl der unter Vertrag genommenen Leistungserbringer in Wohnort- oder Arbeitsplatznähe für die Versicherten sind. Zusätzlich werden die Merkmale „Angebote zur Prämiengestaltung“ und „monatlicher Preisaufschlag auf die Grundprämie“ in die Analyse aufgenommen. Mithilfe einer Limit Conjoint-Analyse im Segment der Studenten und Absolventen $^{51}$ schätzt Scheffold u.a. die Teilnutzenwerte sowie die relative Wichtigkeit der vier Merkmale. ${ }^{52}$ Die Ergebnisse zeigen, „dass die Qualität der Ärzte und Krankenhäuser, mit denen eine Krankenkasse Versorgungsverträge hat, sowie die Höhe des monatlichen Prämienaufschlags im Vergleich zur Prämiengestaltung und der Anzahl der unter Vertrag genommenen Ärzte und Krankenhäuser für die Höhe des Gesamtnutzens des Leistungsangebotes einer gesetzlichen Krankenversicherung besonders wichtig sind. " ${ }^{53}$ Die Qualität und der monatliche Preisaufschlag gehen in die Entscheidung der Versicherten mit einer durchschnittlichen Wichtigkeit von jeweils 37 Prozent ein. Die Anzahl der Leistungserbringer und die Prämiengestaltung weisen dagegen eine deutlich geringere Wichtigkeit von durchschnittlich 17 bzw. 9 Prozent auf. $^{54}$

Die Errungenschaften der gesetzlichen Krankenversicherung haben für die Versicherten einen hohen Wert. Für Patienten ist es bislang selbstverständlich, dass sie ihre Ärzte und Kliniken eigenständig aussuchen können und ihnen der Zugang zum Gesundheitssystem in fast allen Bereichen offen steht. Diese Freiheiten geben GKV-Kunden nur sehr ungern auf. Alternative Versicherungsverträge, die Teile der bisherigen Freiheiten einschränken, werden erst bei entsprechend hohen Beitragsnachlässen akzeptiert. Die Zahlungsbereitschaft für Zusatzangebote ist im Vergleich deutlich geringer. Eine mögliche Erklärung liefert der „Besitzstandseffekt“. Demnach haben Menschen eine Verlustaversion, d.h. der „Schmerz“ über Verluste ist deutlich höher als die „Freude“ über Zugewinne. ${ }^{55}$ Dies drückt sich auch in den jeweiligen Zahlungsbereitschaften und Kompensationsforderungen aus.

Die vorliegenden Conjoint-Analysen, die sich mit den Teilnutzen einzelner (zum Teil fiktiver) Krankenkassenmerkmale auseinandersetzen, stellen eine wichtige Ergänzung zu den bisherigen Untersuchungen des Konsumentenverhaltens in der gesetzlichen Krankenversicherung dar. Anstatt die Wichtigkeit einzelner Angebote direkt abzufragen, werden die Wahlentscheidungen der Versicherten simuliert und der Nutzen spezieller Merkmale mithilfe statistischer Verfahren berechnet. Auf diese Weise lassen sich die Probleme der „Anspruchsinflation“ umgehen und die Präferenzen der Versicherten in Untergruppen ermitteln. Die Nachteile liegen in dem vergleichsweise aufwändigen Untersuchungsdesign und der geringen Anzahl der berücksichtigungsfähigen Merkmale. Um die Studienteilnehmer nicht zu überfordern, sollten sich Paarvergleiche auf wenige Merkmale beschränken.

\section{Zusammenfassung und Fazit}

Auch über zehn Jahre nach der Neuausrichtung des Kassenwettbewerbs nehmen die Versicherten erst wenige Unterschiede zwischen den Krankenkassen wahr. Der Ideenwettbewerb um die besten Versorgungskonzepte konnte sich unter den bisherigen Rahmenbedingungen nur in Teilbereichen entwickeln. Die Wechselbewegungen sind daher vor allem auf die Beitragssätze der Kassen zurückzuführen; Leistungs- und Serviceangebote spielten nur eine 
untergeordnete Rolle. Das Interesse an einem Kassenwechsel ist insgesamt gering.

Trotz dieser mangelnden Differenzierung im Leistungsbereich sehen die Versicherten in ihrer Krankenkasse nicht länger nur den „zahlenden Dritten“, sondern fordern ein stärkeres Engagement bei der Beratung und Betreuung von Patienten und in der Koordination medizinischer Leistungen. Es ist daher zu erwarten, dass sich mit der Einführung des Morbi-RSA und den neu geschaffenen Möglichkeiten der Vertrags- und Tarifgestaltung die Wettbewerbsdynamik in der GKV zukünftig erhöht. Sofern es Krankenkassen gelingt, sich als aktive „Player“ im Gesundheitswesen zu positionieren und Leistungs- und Serviceangebote hervorzubringen, die zur Verbesserung der Qualität und Wirtschaftlichkeit der medizinischen Versorgung beitragen, kann auch mit einem stärkeren Interesse der Versicherten gerechnet werden. Die Gesundheit gilt als wichtigstes Gut eines Menschen. Wer dazu beiträgt, dieses Gut zu erhalten oder es (bestmöglich) wiederherzustellen, dem wird vermutlich auch eine entsprechend hohe Aufmerksamkeit zuteil. Vor diesem Hintergrund besitzt die gesetzliche Krankenversicherung das Potenzial sich zu einem „High Involvement-Produkt" zu entwickeln. Eine ähnliche Prognose stellten auch schon Andersen/ Schwarze (1996) zu Beginn der freien Kassenwahl auf: „Auf eine Phase des Abwartens wird eine Phase des verstärkten Kassenwechsels folgen bis es zu einer Entsprechung von Präferenzstrukturen und Angebotsmodalitäten kommt. " 56 Sieht man von den beitragsgetriebenen Wechselwellen aus den ersten Jahren der freien Kassenwahl ab, dann steht der GKV eine Phase des verstärkten und leistungsgetriebenen Kassenwechsels vermutlich erst noch bevor.

In einem Wettbewerb ist der Anbieter im Vorteil, der weiß, wie die Nachfrager auf spezielle Angebote reagieren, und der dieses Wissen nutzt, um die Wünsche der Kunden bestmöglich zu erfüllen. Zu den Gründen für einen Kassenwechsel und einen Kassenverbleib liegen erste Befragungsergebnisse vor. Auch die Wichtigkeit und der Nutzen bestimmter Kassenmerkmale und -angebote wurden empirisch untersucht.

Über die Entscheidungsprozesse der Versicherten und die Bindung von GKV-Kunden ist dagegen noch relativ wenig bekannt. Zwar wurden mit Hilfe von Conjoint-Analysen die Wahlentscheidungen von gesetzlich Versicherten unter Einbeziehung des Nettonutzens simuliert. Ein ganzheitliches Modell, das neben dem Nettonutzen weitere Determinanten der Kundenbindung einbezieht, fehlt bislang allerdings. Darüber hinaus ist unklar, in welchem Maße die verfügbaren Instrumente das Verhalten der Versicherten bestimmen; unter der Annahme der „Anspruchsinflation“ gibt eine direkte Abfrage von Wichtigkeiten die tatsächliche Bedeutung von speziellen Merkmalen und Angeboten vermutlich nur sehr ungenau wider. Zukünftige Forschungsarbeiten können an diesen Punkten ansetzen.

\section{Fußnoten:}

1 Vgl. Weber (2009)

2 Vgl. Braun et al. (2006), S. $16 \mathrm{ff}$.

3 Vgl. Andersen/Schwarze (1999), S. 29 f.; MSR Consulting Group (2003),

S. 13; Töpfer/Opitz (2008); Sander/Kade-Lamprecht (2008), S. 11 f.

4 Braun et al. (2006), S. 19.

5 Vgl. Schaaf/Sander (2008), S. 11.

6 Vgl. Meyer (2009), S. 560

7 Vgl. Zok (2009), S. 2.

8 Vgl. Töpfer/Opitz (2009), S. 21.

9 Vgl. ASSEKURATA Assekuranz Rating Agentur GmbH (2009), S. 17. 10 Meyer (2009), S. 560.

11 Vgl. ASSEKURATA Assekuranz Rating Agentur GmbH (2009), S. 135.

12 Vgl. Schaaf/Sander (2008), S. 11.

13 Vgl. Braun et al. (2006), S. 17 f.; Greß et al. (2008b), S. 40 f.

14 Zok (2006), S. 5

15 Im Winter 2007/2008 wurden von knapp 21 Prozent der Befragten zwei oder mehr Unterschiede genannt; im Winter 2008/2009 lag die Quote nur noch bei etwa dreizehn Prozent. Vgl. dostal \& partner managementberatung gmbh (2009), S. $27 \mathrm{f}$.

16 Vgl. dostal \& partner management-beratung gmbh (2009), S. 30 f.

17 Vgl. Landmann (2009).

18 Vgl. Korehnke Kommunikation (2009), S. 7 f

19 Vgl. Braun et al. (2006), S. 22 ff.

20 Vgl. Continentale Krankenversicherung a.G. (2002), S. 24 ff.

21 Continentale Krankenversicherung a.G. (2002), S. 26.

22 Vgl. Felder (2007), S. 218 f.

23 Vgl. Felder (2007), S. 219

24 Vgl. Paquet/Stein (2008), S. 276 ff.

25 Vgl. Korehnke Kommunikation (2009), S. 5.

26 Vgl. Braun et al. (2006), S. 18; Greß et al. (2008b), S. 41 f.

27 Vgl. Töpfer/Opitz (2009), S. 8; zu ähnlichen Ergebnissen vgl. auch Braun/Streich (2003), S. 74.

28 Vgl. Töpfer/Opitz (2009), S. 9

29 Vgl. Braun et al. (2006), S. 19; Greß et al. (2008a), S. 15.

30 Vgl. Höppner et al. (2004), S. 28 ff.; Braun et al. (2006), S. 20 ff.; Greß et al. (2008a), S. $13 \mathrm{ff}$.

31 Vgl. Braun et al. (2006), S. 22.

32 Vgl. Sachverständigenrat für die Konzertierte Aktion im Gesundheitswesen (1995), S. 141.

33 Braun/Streich (2003), S. 71.

34 Vgl. Bode/Bühren (2004), S. 50

35 Bode/Bühren (2004), S. 53.

36 Vgl. Bode/Bühren (2004), S. 53

37 Kartte/Neumann (2008), S. 10

38 Zok (2009), S. 7.

39 Vgl. Zok (2009), S. 8.

40 Töpfer/Opitz (2009), S. 15.

41 Vgl. Gruner + Jahr AG \& Co KG (2007), S. 22; BKK Bundesverband (2007), S. 2; Meyer (2009), S. 560; Landmann (2009), S. 213.

42 Vgl. Dichtl/Müller (1986).

43 Vgl. z.B. Matzler/Sauerwein/Stark (2004).

44 ASSEKURATA Assekuranz Rating Agentur GmbH (2009), S. 21

45 Zum Vorgehen einer Conjoint-Analyse siehe z.B. Backhaus et al. (2006), S. 557 ff.; Homburg/Krohmer (2006), S. 407 ff.

46 Vgl. Nolting et al. (2006)

47 Nolting et al. (2006) berechnen diesen Wert wie folgt (S. 39): „Bei einem GKV-Mitglied mit durchschnittlichen beitragspflichtigen Einnahmen (knapp $22.000 €$ ) und bei einem Beitragssatz von 14 Prozent (bzw. 7 Prozent für das Mitglied allein) entsprechen 17,6 Prozent Reduktion einem jährlichen Beitragsnachlass von etwa $260 € . "$

48 Die Studienergebnisse wurden veröffentlicht in Becker/Zweifel (2006) und Becker/Brändle/Zweifel (2007).

49 Vgl. Greß et al. (2008b), S. $56 \mathrm{ff}$

50 Vgl. Scheffold (2008); Cassel/Nihalani (2008).

51 Anzahl der Probanden: 165; Erhebungszeitraum: April bis Juni 2005

52 Vgl. Scheffold (2008), S. $191 \mathrm{ff}$.

53 Cassel/Nihalani (2008), S. 432

$54 \mathrm{Vgl}$. Scheffold (2008), S. 219.

55 Vgl. Diller (2000), S. 133

56 Andersen/Schwarze (1996), S. 822 f. 


\section{Literatur:}

Andersen, Hanfried/Schwarze, Johannes (1996): Die freie Kassenwahl - ein gesellschaftliches Experiment: Ökonomische und soziologische Analysen individueller Entscheidungsprozesse und Präferenzbildungen, Zeitschrift für Sozialreform, 42, Nr. 11/12 (1996), 810-825.

Andersen, Hanfried H./Schwarze, Johannes (1999): Der VersichertenReport. Methodische und inhaltliche Aspekte einer standardisierten Versichertenbefragung. Die Novitas Befragung 1998, Veröffentlichungsreihe des Berliner Zentrum Public Health, 99-2.

ASSEKURATA Assekuranz Rating Agentur GmbH (2009): KrankenkASSen im Kundenspiegel. Was die Versicherten erwarten, Studie, Juli 2009.

Backhaus, Klaus et al. (2006): Multivariate Analysemethoden. Eine anwendungsorientierte Einführung, Elfte, überarbeitete Auflage. Berlin, Heidelberg: Springer.

Becker, Karolin/Brändle, Angelika/Zweifel, Peter (2007): Das Discrete-Choice-Experiment, In Böcken, Jan et al. (Hrsg.): Neue Versorgungsmodelle im Gesundheitswesen. Gestaltungsoptionen und Versichertenpräferen zen im internationalen Vergleich, 38-151.

Becker, Karolin/Zweifel, Peter (2006): Neue Formen der ambulanten Versorgung: Was wollen die Versicherten? Ein Discrete-Choice-Experiment, In Böcken, Jan et al. (Hrsg.): Gesundheitsmonitor 2006. Gesundheitsversorgung und Gestaltungsoptionen aus der Perspektive von Bevölkerung und Ärzten, Bertelsmann Stiftung, 272-344.

BKK Bundesverband (2007): BKK Faktenspiegel. Schwerpunktthema Krankenkassenwahl, BKK Faktenspiegel: Der Newsletter für Gesundheitsdaten, September 2007, http://www.bkk.de/faktenspiegel (Verfügbarkeitsdatum: 23.06.2009)

Bode, Ingo/Bühren, Patrick (2004): Mehr als Markt und Bürokratie. Krankenkassen im Bild von Versicherten und Mitarbeitern, Gesundheitsund Sozialpolitik, 58, Nr. 1-2 (2004), 48-57.

Braun, Bernard et al. (2006): Barrieren für einen Wechsel der Krankenkasse: Loyalität, Bequemlichkeit, Informationsdefizite? In Böcken, Jan et al. (Hrsg.): Gesundheitsmonitor 2006. Gesundheitsversorgung und Gestaltungsoptionen aus der Perspektive von Bevölkerung und Ärzten, Bertelsmann Stiftung, 11-31.

Braun, Bernard/Streich, Waldemar (2003): Gutes Beitrags-Leistungs-Verhältnis oder Sicherheit - was wollen die Bürger von ihrer Krankenkasse? In Böcken, Jan/Braun, Bernard/Schnee, Melanie (Hrsg.): Gesundheitsmonitor 2003. Die ambulante Versorgung aus Sicht von Bevölkerung und Ärzteschaft, Bertelsmann Stiftung, 71-84

Cassel, Dieter/Nihalani, Katrin (2008): Präferenzgerechte Versorgungsangebote zur Kundenbindung im Kassenwettbewerb, In Funk, Lothar (Hrsg.): Anwendungsorientierte Marktwirtschaftslehre und Neue Politische Ökonomie. Wirtschaftspolitische Aspekte von Strukturwandel, Sozialstaat und Arbeitsmarkt. Eckhard Knappe zum 65. Geburtstag, Metropolis-Verlag, 421-435

Continentale Krankenversicherung a.G. (2002): Continentale-Studie 2002 Zusatzversicherung und GKV: Die Einstellung der Bevölkerung, http:// www.continentale.de (Verfügbarkeitsdatum: 23.06.2009).

Dichtl, Erwin/Müller, Stefan (1986): Anspruchsinflation und Nivellierungstendenz als meßtechnische Probleme in der Absatzforschung, Marketing - Zeitschrift für Forschung und Praxis, 8, Nr. 4 (1986), 233-236.

Diller, Hermann (2000): Preiszufriedenheit bei Dienstleistungen, Die Betriebswirtschaft, 60, Nr. 5 (2000), 570-587.

dostal \& partner management-beratung gmbh (2009): Vom Desinteresse zur Unterscheidbarkeit: Informationsverhalten und Wahrnehmung der Verbraucher zum Thema Gesetzliche Krankenkassen, dostal-Studie, Mai 2009.

Felder, Stefan (2007): Gesundheitsfonds und Preiswettbewerb, In Göpffarth, Dirk et al. (Hrsg.): Jahrbuch Risikostrukturausgleich 2007, AsgardVerlag, 215-230.

Greß, Stefan et al. (2008a): Kassenwechsel zur Durchsetzung von Versicherteninteressen? Ergebnisse einer repräsentativen Befragung von GKVVersicherten, Soziale Sicherheit, 57, Nr. 1 (2008), 12-17.

Greß, Stefan et al. (2008b): Kassenwechsel als Mechanismus zur Durchsetzung von Versicherteninteressen, In Braun, Bernard et al. (Hrsg.): Einfluss nehmen oder aussteigen. Theorie und Praxis von Kassenwechsel und Selbstverwaltung in der Gesetzlichen Krankenversicherung, 19-89.

Gruner + Jahr AG \& Co KG (2007): Krankenkassen und Gesundheitsreform stern TrendProfile, August 2007, http://www.gujmedia.de (Verfügbarkeitsdatum: 23.06.2009).
Homburg, Christian/Krohmer, Harley (2006): Marketingmanagement. Strategien -Instrumente - Umsetzung - Unternehmensführung, 2. Auflage. Wiesbaden: Gabler.

Höppner, Karin et al. (2004): Kassenwettbewerb: Motive für einen Kassenverbleib, In Böcken, Jan/Braun, Bernard/Schnee, Melanie (Hrsg.): Gesundheitsmonitor 2004. Die ambulante Versorgung aus Sicht von Bevölkerung und Ärzteschaft, Bertelsmann Stiftung, 24-34.

Kartte, Joachim/Neumann, Karsten (2008): Der Gesundheitsmarkt. Sicht der Bürger - Strategien der Anbieter, Studie, Roland Berger Strategy Consultants, Germany.

Korehnke Kommunikation (2009): Kassenbindung der gesetzlich Krankenversicherten. Ergebnisse einer Repräsentativbefragung, Studie, http:// www.korehnkekommunikation. (Verfügbarkeitsdatum: 23.06.2009).

Landmann, Juliane (2009): Krankenkassenwettbewerb um Versicherte: Diesmal ohne Beitragssatz, In Böcken, Jan/Braun, Bernard/Landmann, Juliane (Hrsg.): Gesundheitsmonitor 2009. Gesundheitsversorgung und Gestaltungsoptionen aus der Perspektive der Bevölkerung, Bertelsmann Stiftung, 203-220.

Matzler, Kurt/Sauerwein, Elmar/Stark, Christian (2004): Methoden zur Identifikation von Basis-, Leistungs- und Begeisterungsfaktoren, In Hinterhuber, Hans H./Matzler, Kurt (Hrsg.): Kundenorientierte Unternehmensführung, 4. Auflage., 315-339.

Meyer, Jörg (2009): BKK Imagemonitor 2008, Die BKK, 96, Nr. 10 (2008), 557-561.

MSR Consulting Group (2003): Wechselverhalten in der Gesetzlichen Krankenversicherung. Ergebnisse einer repräsentativen Studie, Ergebnisbericht, Köln, August 2003.

Nolting, Hans-Dieter et al. (2006): Nutzen, Kosten, Präferenzen - Wissen was der Bürger will! 4. Delphi-Studie zur Zukunft des Gesundheitswesens, Janssen-Cilag GmbH.

Paquet, Robert/Stein, Martin (2008): GKV-Mitglieder: preissensibel und leistungsbewusst, Die BKK, 96, Nr. 05 (2008), 274-280.

Sachverständigenrat für die Konzertierte Aktion im Gesundheitswesen (1995): Gesundheitsversorgung und Krankenversicherung 2000. Mehr Ergebnisorientierung, mehr Qualität und mehr Wirtschaftlichkeit, Baden-Baden: Nomos.

Sander, Michael/Kade-Lamprecht, Evelyn (2008): Kundenbeziehungsmanagement entscheidet den Wettbewerb in der GKV, Fokusvortrag zur 3 GKV-Managementfachtagung am 29. April 2008 in Hamburg, http:// www.terraconsult.de (Verfügbarkeitsdatum: 23.06.2009).

Schaaf, Michael/Sander, Michael (2008): Marketing und Strategie im Gesundheitswesen. Diskussionsbeitrag zur Bewältigung des Wandels speziell im Sektor GKV Gesetzliche Krankenversicherung, White Paper, April 2008, Veröffentlicht von: TCP Terra Consulting Partners GmbH.

Scheffold, Katrin (2008): Kundenbindung bei Krankenkassen. Eine marketingorientierte Analyse kassenindividueller Handlungsparameter bei selektivem Kontrahieren auf dem GKV-Versorgungsmarkt, Berlin: Duncker \& Humblot.

Töpfer, Armin/Opitz, Frank (2008): Benchmarking mit dem Versichertenbarometer - Kundenzufriedenheit und -bindung im deutschen Krankenkassenmarkt, In Töpfer, Armin (Hrsg.): Handbuch Kundenmanagement: Anforderungen, Prozesse, Zufriedenheit, Bindung und Wert von Kunden, Heidelberg, 541-553.

Töpfer, Armin/Opitz, Frank (2009): M+M Versichertenbarometer 2009. Kundenzufriedenheit und -bindung im Urteil der Versicherten, Studienbericht der Management + Marketing Consulting GmbH, März 2009

Weber, Guido W. (2009): Wechseldynamik, Wechslerprofile und Motive der Kassenwahlentscheidung. Empirische Befunde zum Konsumentenverhalten in der gesetzlichen Krankenversicherung, Gesundheits- und Sozialpolitik, 63, Nr. 6 (2009), 11-21.

Zok, Klaus (2002): Erwartungen der Versicherten an die Gesetzliche Krankenversicherung, Arbeit und Sozialpolitik, 56, Nr. 3-4 (2002), 29-35.

Zok, Klaus (2006): Beitragssatzkenntnis und Wechselbereitschaft in der GKV. Ergebnisse von Repräsentativumfragen bei GKV-Mitgliedern im Zeitverlauf, WIdO-monitor, 3, Nr. 2 (2006), 1-7.

Zok, Klaus (2009): Erwartungen an die GKV nach Einführung des Gesundheitsfonds. Ergebnisse einer Repräsentativ-Umfrage unter 3.000 GKVVersicherten, WIdO-monitor, 6, Nr. 1 (2009), 1-8. 